\title{
THE USE OF VEHICLE DATA IN ADAS DEVELOPMENT, VERIFICATION AND FOLLOW-UP ON THE SYSTEM
}

\author{
J. Orlovska ${ }^{1, \otimes}$, C. Wickman ${ }^{1,2}$ and R. Soderberg ${ }^{1}$ \\ ${ }^{1}$ Chalmers University of Technology, Sweden, ${ }^{2}$ Volvo Cars, Sweden \\ $\square$ orlovska@chalmers.se
}

\begin{abstract}
Advanced Driver Assistance Systems (ADAS) require a high level of interaction between the driver and the system, depending on driving context at a particular moment. Context-aware ADAS evaluation based on vehicle data is the most prominent way to assess the complexity of ADAS interactions. In this study, we conducted interviews with the ADAS development team at Volvo Cars to understand the role of vehicle data in the ADAS development and evaluation. The interviews' analysis reveals strategies for improvement of current practices for vehicle data-driven ADAS evaluation.
\end{abstract}

Keywords: data-driven design, complex systems, case study, design evaluation, product development

\section{Introduction}

Advanced Driver Assistance Systems (ADAS) are designed to support driving tasks. These systems provide longitudinal control of a vehicle through accelerating or braking in various traffic conditions, and/or lateral control through administering steering assistance. However, the usage of these systems does not depend solely on system performance. Today these systems are not able to support the driver in all driving situations. ADAS functions have a number of performance limitations in different traffic, weather and road conditions. For example, bad visibility on the road can lead to deactivation of ADAS, while the absence of lane markings on the roadway can interrupt ADAS support of steering function. These examples illustrate the fact that the driving context (for ADAS the driving context is defined as the aggregation of traffic, road, and weather conditions) plays a crucial role in this communication. For that reason, the contextual information supporting every event of the driving activity is an essential factor for comprehensive ADAS evaluation (Carta et al., 2011; Angelini et al., 2018).

Thus, driver decisions regarding the activation/deactivation of support systems are not only subjective but are influenced by the system performance and driving conditions at the moment of decision making. If a driver attempts to activate the system in a driving context that does not comply with the design intent, the system performance can become unstable, resulting in rejection from the user side. In such cases, evaluation of the system performance without acknowledgment of driver behavior and the driving context in a particular situation will prevent developers from gaining a comprehensive understanding of the underlying issues regarding driver-system interaction. Thus, 
to be able to evaluate driver behavior in various driving situations, all context parameters affecting ADAS performance need to be considered.

Although there is a strong interrelation between driver and system in ADAS systems, in practice the driver behavior and the system performance is often evaluated in isolation. Such an approach is primarily defined by the feasibility of data resources, human expertise, and adopted traditions within a particular field or company (Orlovska et al., 2019). In the automotive industry, the user-related vehicle data is usually poorly developed and less utilized in the user behavior evaluation process in comparison to other fields. Therefore, quantitative methods are often substituted by traditional qualitative methods such as in-depth interviews, observations, surveys.

At the same time, the continuously increasing in-vehicle connectivity opens new capabilities for obtaining new objective usability data. Previously conducted studies based on objective data (Benmimoun et al., 2013; Neale et al., 2005; Fridman et al., 2019) demonstrated the great potential of utilizing vehicle data for diverse user-related studies. This user-related objective data is generated with high velocity and in real-time. Along with the system performance assessment, the vehicle data analysis gives us the possibility for context-aware user behavior evaluation and indicates how well the user understands the system functionality. Objective data also offers the ability to determine certain trends in user behavior, as well as to identify specific use errors, the usage of a particular function, or the degrading of usage of a function over time (Orlovska et al., 2019). Additionally, the ongoing research examining the quality of vehicle data and its applicability has a positive effect on the feasibility of this data, which has been gradually improving over recent years.

It is also important to mention that verifying of semi-automated systems become a more and more complex task, since the level of ADAS automation increases. To avoid potential safety hazards, AI and machine learning will become critical for developing systems with a high level of automation (ISO/PAS 21448, 2019). This means that the data and applications for data utilization will become more complicated in the near future. Thus, automotive OEMs need to continually improve the quality of data and the methods they use to make transition steps easier and be able to meet future requirements.

In this study we conducted semi-structured interviews with the ADAS development team at a Swedish leading automotive company (OEM), aiming to understand what means and methods are used today for ADAS evaluation and what role the vehicle sensors data plays in current practices. Analysis of the interview data allowed us to uncover how and in what applications vehicle data is used throughout ADAS development, verification, and usage evaluation. This knowledge helps to discuss the effectiveness of using vehicle data and to propose possibilities for improvement of current practices for data-driven ADAS evaluation.

This paper is structured as follows: Section 2 presents the relevant background for the study; Section 3 describes the methodology; Section 4 presents the main findings of the interviews; Section 5 discusses the effectiveness of vehicle data utilization and the methods used for ADAS assessment; Section 6 provides conclusions and recommendations for further research.

\section{Background}

ADAS systems evaluated in this study refers to levels 1-2 of the SAE automation (2018). According to SAE International (2018), there are three primary actors in driving with ADAS: the driver, the ADAS, and vehicle systems and components influencing ADAS performance. Moreover, all interactions between the driver and the system happen in the dynamic driving context. The driving context is the summary of external factors that affect driver behavior while using the evaluated system (Zhai et al., 2018), which highly depends on the evaluated objectives and needs to be set accordingly. For ADAS evaluation, the driving context is defined as the aggregation of traffic, road, and weather conditions that in association encourage or discourage the usage of ADAS. Thus, the driving context plays a central role in interactions between the driver and ADAS. Due to its limitations, ADAS does not perform equally well in all driving conditions. Therefore, to be able to evaluate driver behavior, all context parameters affecting ADAS performance need to be considered. The ADAS interrelating factors are presented in Figure 1. As can be seen in this figure, the ADAS performance depends on the performance of additional in-vehicle systems, which contribute to ADAS performance, and on driver 
behavior. The driver behavior, in turn, depends on the cumulative abilities of the technical solutions supporting the ADAS performance in the current driving situation. The driving context affects driver and system performance. Both need to consider the ADAS limitations regarding the driving context that the system can handle.

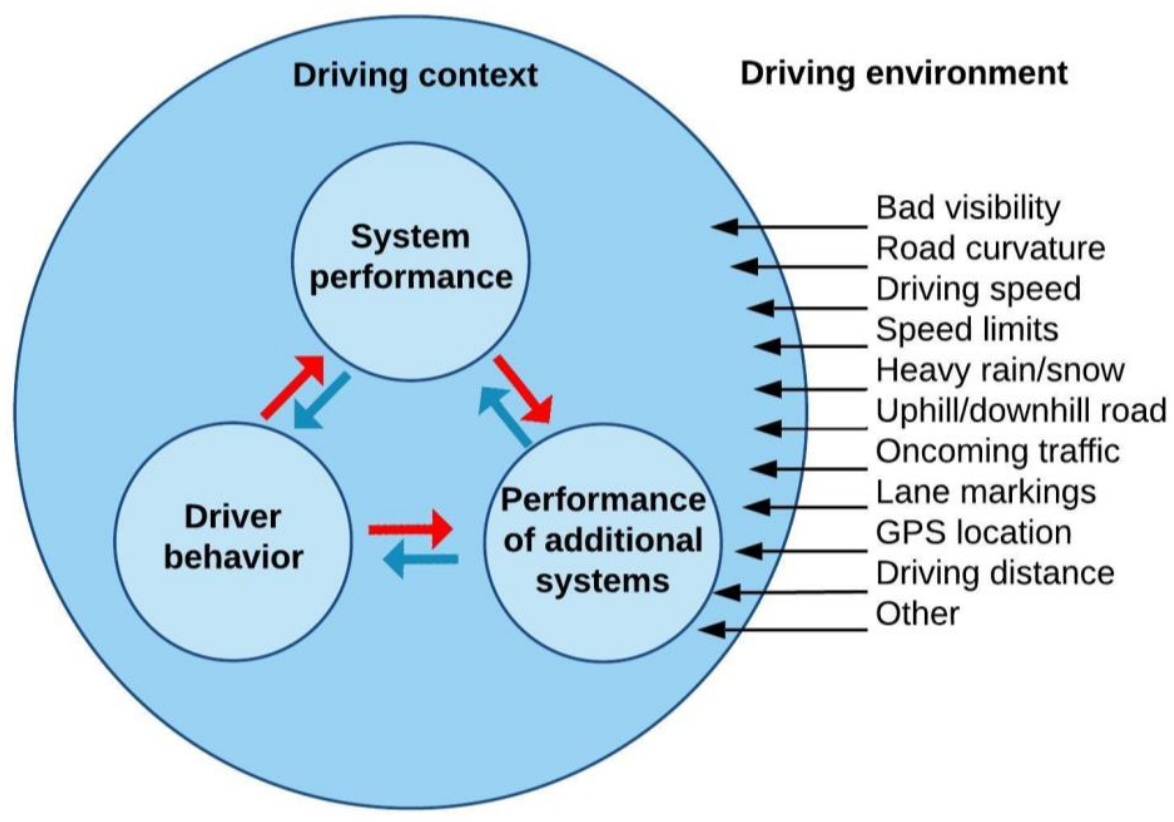

Figure 1. ADAS interrelating factors

As can be seen, there is a strong interrelation between the driver and system in ADAS. However, despite the need for a holistic approach in ADAS evaluation that would consider the driver, the system, and the dynamic driving context altogether, in many cases the system and driver evaluation are still carried out in isolation. If the advantages of using vehicle sensors' data for system performance verification are apparent, the means and methods for driving context assessment and driver behavior evaluation are still developing. Several studies have emphasized the effect of driving context on driving behavior, as well as on drivers' interactions with invehicle systems (Liang et al., 2016; Ahlström et al., 2018; Ahmed and Ghasemzadeh, 2018; Papazikou et al., 2017; Zhai et al., 2018). Liang et al. (2016) underlined the importance of driving context analysis for detecting abnormal driver behavior, aiming to quantify the risks associated with various driver behaviors. Zhai et al. (2018) emphasized the importance of context-aware driver behavior evaluation, showing that the integrating of driving context provides reliable results regarding driver behavior evaluation on the road. According to Papazikou et al. (2017), the driving context is one of the most important factors for user behavior evaluation. Both papers conclude that the driving context might affect driver behavior, both positively and negatively. Studies such as Ahlström et al. (2018) and Ahmed and Ghasemzadeh (2018) have emphasized the effect of contextual factors on driver behavior, saying that the driving context must be considered over the full spectrum of its complexity. Some researchers have focused on the development of methods for automated driving event recognition (Mitrovic, D., 2005; Leakkaw and Panichpapiboon, 2018). Automated driving event recognition contributes to the ability for automated analysis of the effect of driving context on both system and driver performance.

The understanding of the need for holistic ADAS evaluation stimulated the development of technical solutions for Naturalistic Driving (ND) studies that became helpful for understanding driving behavior in a driving context, investigating the complexity of driver and system interaction in ADAS (Benmimoun et al., 2013; Neale et al., 2005; Fridman et al., 2019). ND study refers to a study where a strict experimental design does not constrain the data collection, and where the data is gathered in a natural driving context and under various driving conditions that closely resemble real-driving 
situations (Fridman et al., 2019). In this type of study, a large number of vehicles are equipped with data acquisition systems that continuously and inconspicuously register driver behavior, vehicle maneuvers, and external conditions over more extended periods. Vehicle data, as the primary source of ND data, enables unobtrusive driver behavior evaluation in a time-efficient and reliable way to help to determine specific usability problems and assess their severity.

The studies described above highlight the evidence that vehicle data can be used for the complex assessment of driver-system interaction while considering the spectrum of contextual factors affecting this interaction. Today, when the number of vehicles with built-in telematics systems is only increasing, the utilization of on-board diagnostics looks like the most promising approach for vehicle support systems evaluation. Without using this approach, the developers often struggle to obtain a comprehensive understanding of the interrelating contextual factors and the range of their effects on driver behavior and system performance.

\section{Method}

In this research we designed an exploratory case study that included case design, data collection methods, and approaches to data analysis (Yin, 2013). The goal of this study was to investigate how the data flow is organized in ADAS development and evaluation. We performed semi-structured interviews with follow-up questions, with the aim of stimulating further discussion on the investigated topic. Interviews as a data collection method are a valid and reliable choice (Beggiato and Krems, 2013), seeking to obtain knowledge about the verification methods used in the ADAS development lifecycle and the extent the vehicle data is utilized in these methods.

During the interviews we documented the methods the departments use to test system performance and usage processes. The focus of the interviews was the extent to which vehicle data is used in these approaches.

\subsection{Participants}

The in-depth semi-structured interviews included the OEM employees involved in the development, verification, and promotion of ADAS systems. All participants are long-term employees with key positions in the company regarding the evaluated driver support systems. All participants were personally involved in defining ADAS functionalities and were responsible for the development, verification, and promotion of these functions. The interview study consisted of 5 participants, four males and one female. All participants recruited are part of the ADAS development, verification and marketing teams and have leading positions in ADAS development (see Table 1).

Table 1. An overview of the interviewees

\begin{tabular}{|l|l|}
\hline Interviewee & Affiliation \\
\hline Interviewee TR1 & Product development/Product owner \\
\hline Interviewee TR2 & Product development/Function owner \\
\hline Interviewee TR3 & Product development/Attribute leader \\
\hline Interviewee TR4 & Product development/Attribute leader \\
\hline Interviewee TR5 & Marketing/Business owner \\
\hline
\end{tabular}

\subsection{Study design and procedure}

Semi-structured interviews were conducted at the OEM's premises during working hours and at a time convenient for the participants. The interview study was conducted between November 2018 and January 2019. All interviews were audio-recorded with the participants' consent. The structure of the interview and the interview questions were based on four main parts: ADAS design and intent; ADAS verification methods; Methods for usage evaluation; Re-design process. Thus, a set of open-ended questions was developed, based on these four themes. During the interviews we asked the same questions of all participants involved in the study. Briefly describing the protocol design at the beginning of each interview, the questions were quite open and general, for example:

- What stages do you go through to verify ADAS design and development? 
The subsequent questions narrowed the interest in understanding the type of data the evaluation is based on for vehicle data in these stages, for example:

- How do you verify the system performance in the pre-production phase?

- What methods are used?

- What data is collected?

- How do you use this data?

The interview was conducted using the developed topic guide. However, the interview was not limited to the sample questions, and the participants were encouraged to elaborate on their experiences and provide more descriptive insights. All sessions were conducted in English, where each session lasted about 40-50 minutes. The interview data in this study, regarding all accumulated data points, was collected with the informed consent of all participants. The retrieval, storage and processing of the collected data was accomplished strictly in accordance with the European general data protection regulations (GDPR). The data was processed confidentially, and all participants' identities were kept anonymous.

\subsection{Interview analysis}

All interviews were carefully transcribed verbatim, coded and analyzed with the help of qualitative data analysis software NVivo 12 (NVivo, 2019). Two independent coders examined the first transcript. According to the interview structure, the researchers identified different themes and their meanings within the categories. In the next step the themes were reviewed and discussed in order to determine coherence and minimize subjective discrepancy. Consequently, the interviews were coded by each researcher separately and a final session was held, where the open questions and themes were discussed to review the quality of the coding.

\section{Findings}

In the course of this study two ways of collecting vehicle data were identified. The first way is the data collection acquired through the ADAS software code. This process of data acquisition is organized by ADAS software developers to record specific data needed for internal ADAS performance verification by developers. To make it easier, in this study we will call this type of vehicle data internal.

Another source of vehicle data is the ND study, where the CAN-bus and GPS location data are collected as part of OEM's extensive project. In this paper, we will call this type of data external, due to the additional acquisition system that every test vehicle needs to be instrumented with.

This section presents the main results of the analysis of the interviews focusing on the utilization of vehicle data in three domains: system performance, driving context, and driver behavior. Analysis of the interview data helped to uncover how and in what stages vehicle data is used for ADAS verification and follow up processes.

\subsection{System performance}

According to the interview data, in the process of ADAS performance verification, three phases are named as important: (1) internal verification by ADAS developers; (2) complete vehicle test and (3) follow up on ADAS performance with real users.

The internal verification by ADAS developers includes several activities, starting from software verification, unit test verification and internal evaluation in the real environment conducted by developers. At the stage of software verification the code is verified by software methods or on simulators, since there is no vehicle yet. The use of vehicle data starts at the moment when software verification is first moved to test vehicles. For that reason, the software system design implies internal vehicle data collection, which records system performance data to support the ADAS performance verification process on the test vehicle. At the stage of ADAS verification in the real environment, more vehicles instrumented with the new ADAS version are tested. The development team tests ADAS in all the loops, trying to validate the system and describe the limits within which the system works well. In this first phase, the internal vehicle data, together with the video records of different driving events, are extensively analyzed. 
Approximately a year before production the complete vehicle test starts when all systems in the vehicle need to be validated by professional customers. In this phase, engineers from different departments, with a wide range of expertise, perform a complete vehicle assessment to test the compliance of all systems in the vehicle. This evaluation is based on the feedback forms, where professional drivers report all of the issues with vehicle systems and their cooperation in a complete vehicle.

After the production starts, no improvements in system performance in the current version of ADAS can be made. Therefore, only the follow-up process is possible. However, despite the fact that the vehicle pool providing data is expanding, there is not much interest given to the follow-up of ADAS performance. At this stage the ADAS functionalities are considered to be verified and validated within the set limitations. The user feedback on system performance, in the form of eventual complaints about vehicle performance, is collected through the service technicians and distributors, who receive information about all of the technical issues regarding the newly launched system. This input comes back to the developers and every function owner is updated with a list of issues raised often on different markets, different vehicle models, etc. As an additional input, marketing buys data from such external surveys as J.D. Power to get statistics and ratings on ADAS performance.

Thus, the interview analysis revealed that internal vehicle data is the primary data source in the process of ADAS development and verification. This data is heavily used in all stages of ADAS verification, collecting data from all vehicles entering the market.

\subsection{Driving context}

Today, the ADAS functions performance is restrained by a number of limitations connected to the driving context situation. Thus, the driving context has a significant effect on the ability of ADAS functions to support a driver. "If you have a straight road and clear lane markings, ADAS deactivation seldom happens. If it is rainy and it is curvy, and you have poor lane markings, it can happen all the time." The impact of the driving context on system performance is vast, and ADAS developers understand the need to evaluate how ADAS functions work under different driving conditions. Thus, in the pre-production phase, ADAS are tested in various driving contexts to detect and describe all limitations when systems are not stable in their performance.

However, it appeared that the internally collected vehicle data was not able to provide comprehensive contextual information regarding the situations when activation or deactivation happened. One of the principal constraints preventing the evaluation of system performance in different driving contexts is that the data from the internal acquisition system is not time-stamped. This means that the acquisition system works as a counter of system performance events but cannot contribute to the driving situation analysis. As a result, it is not possible to separate data among two driving events and analyze it comparing one driving event to another. "We specified [internal vehicle data] a couple of years before the first vehicle went to production. So, we can look at what we thought was important at that time [...] It is really difficult to draw any conclusion on that [meaning driving context]. It is mainly to see if they have used it or not."

To overcome the limitations of the internal vehicle data, ADAS developers collect subjective feedback on how the system reacts to various situations in a dynamic driving context. Developers and professional customers drive thousands of kilometres to test the system in different driving conditions and document all context issues during the test period.

In the post-production stage, new possibilities to obtain reliable vehicle data appear. The driving context can be assessed through the external ND data, collected by the OEM in the ND study. Despite recent research (Orlovska et al., 2020) that showed that the external vehicle data collection in a real driving environment organized by OEMs is a reliable source to follow up system performance in various driving contexts, this data is not extensively used by ADAS developers. "We haven't done that much with it [external vehicle data]. But we rather have our own data recorder, and that is what we have."

Summarising the above for the driving context assessment, developers still give priority to their internal data source and combine it with other subjective evaluation methods, such as feedback from expeditions or video recording of system performance in various driving conditions.

\subsection{Driver performance}


The acceptance and usage of ADAS has great importance for ADAS development, stimulating the driver in-the-loop evaluation. During the pre-production phase, when the developers internally test the system in the real environment, more focus is given to the validation of the technical capabilities of the designed system, and less attention is paid to the evaluation of the driver's ability to understand and collaborate with the technology. As was stated in the interviews, the pre-production verification phase is not where driver behavior is evaluated. However, all interviewees underlined the importance of early notice of driver reaction on ADAS functionalities. Therefore, during the system performance tests, ADAS developers try to set themselves "in a position where the customer will end up in" and perform expeditions "more or less as the customers" to receive early feedback on possible user issues with the systems.

Subsequently, when the complete vehicle test starts, the professional customers continually drive all the loops to provide subjective input on any detected issues that drivers can meet or anticipate in the real environment. "They really try not to focus on how the technology works, but on how the customer perceives this. [...] So we can call it some kind of internal review, as well as about using this."

As a parallel activity, UX-UI engineers organize studies investigating how the systems and the functionalities they provide are interpreted by the drivers. A part of these clinics is focused on the HMI interaction and how it works for the drivers. Do drivers "understand how to use the function, or do they need like a 50-page manual to read before they can sit in the car and use the system?"

As previously mentioned, at the stage of complete vehicle evaluation, internal vehicle data is also collected. However, the internal data used by developers for system performance evaluation is not suitable for the assessment of driver behavior. The internal data collected by developers is able to assess driver behavior at a very general level, indicating the number of activations and the total time ADAS was used. No indications of different use strategies that depend on the driving context can be derived from internal vehicle data only.

When the first vehicles with ADAS appear on the market, the primary focus shifts to the driver evaluation, and the main questions in this phase are how drivers understand, use, and accept the developed ADAS functions. External ND data is useful for this type of evaluation. However, the ND study setup requires time. A number of vehicles need to be instrumented, and several months of data collection needs to be performed before ND data-driven results can be achieved.

Since the ND data is not instantly available and internal data is not valid for driver behavior evaluation, the driver feedback is collected from multiple studies, clinics, and review articles organized within and outside the OEM. These studies are conducted for different purposes and in different setups from the beginning, and the driver feedback is not often connected to the driving context where one or another issue was identified. As a consequence, developers don't have complete results, and often show their uncertainty while talking about the usage of their systems and user opinion. Moreover, the results of different studies are often contradictory, which makes the use of the driver input even more obscure in the re-development process.

However, the developers need to understand how drivers react to their solutions and if their behavior complies with the predefined use scenarios. During the interviews a few participants expressed the opinion that they needed a better input on driver behavior of the evaluated functions, stating "What we perhaps lack knowledge about is how the real customer uses the function. So, that is what we must be better at."

All study participants agreed that the vehicle data needs to be better utilized for driver behavior evaluation. All interviewees also recognized the potential of external ND study data, which more effectively collects data on driver performance in various driving conditions. This data is required to follow up on the driver-system interaction. However, in practice, the ND data is not used to its full capacity, which indicates that the new methods need time to be adopted at the company level.

Summarizing the activities for driver behavior evaluation, it became evident that the major input comes from subjective data collection. The ability of external ND data for driver behavior evaluation is recognized, but the ND study is not yet used to its full capacity.

\subsection{Summary of the results}

Analysis of the interviews revealed that there is currently no systematic approach regarding the use of vehicle data. In general, vehicle data is extensively used for system performance verification, but is 
less used in the driver performance evaluation and driving context assessment. Thus, internal vehicle data is used only for system performance verification. The contribution of internal vehicle data to the understanding of the driving context and driver behavior is very vague. External ND data is not used to its full capacity, despite the great benefits that it can provide in supporting different contexts and use scenarios registration. The benefits of such studies are not yet evident to the developers. The reason could be that the ND study is a relatively new approach for comprehensive vehicle systems evaluation at the company, and therefore requires further development and more time to be accepted as a reliable data source for complete ADAS evaluation. A summary of the data used for ADAS system, driver and driving context verification is presented in Table 2.

Table 2. Data used in different stages of ADAS verification processes

\begin{tabular}{|l|l|l|l|}
\hline & System performance & Driving context & Driver behavior \\
\hline $\begin{array}{l}\text { Unit test } \\
\text { verification }\end{array}$ & $\begin{array}{l}\text { Internal vehicle data } \\
\text { software code }\end{array}$ & $\begin{array}{l}\text { Internal vehicle data } \\
\text { video recording }\end{array}$ & $\begin{array}{l}\text { None (not evaluated by } \\
\text { developers at this stage) }\end{array}$ \\
\hline $\begin{array}{l}\text { Internal } \\
\text { verification by the } \\
\text { development team }\end{array}$ & Internal vehicle data & $\begin{array}{l}\text { Internal vehicle data } \\
\text { feedback from developers } \\
\text { testing ADAS }\end{array}$ & $\begin{array}{l}\text { Feedback from developers } \\
\text { testing ADAS }\end{array}$ \\
\hline $\begin{array}{l}\text { Complete vehicle } \\
\text { test (OEM level) }\end{array}$ & $\begin{array}{l}\text { Internal vehicle data } \\
\text { feedback from } \\
\text { professional customers }\end{array}$ & $\begin{array}{l}\text { Internal vehicle data } \\
\text { feedback from professional } \\
\text { customers }\end{array}$ & $\begin{array}{l}\text { Feedback from } \\
\text { professional customers }\end{array}$ \\
\hline $\begin{array}{l}\text { Follow up on } \\
\text { system(s) with real } \\
\text { customers. }\end{array}$ & $\begin{array}{l}\text { Internal vehicle data, } \\
\text { issues reported through } \\
\text { the service centers, } \\
\text { external surveys such as } \\
\text { J.D. Power }\end{array}$ & $\begin{array}{l}\text { External vehicle data } \\
\text { market intelligence studies } \\
\text { in different markets }\end{array}$ & $\begin{array}{l}\text { External vehicle data } \\
\text { market intelligence studies } \\
\text { with real drivers } \\
\text { detailed questionnaires }\end{array}$ \\
\hline
\end{tabular}

\section{Discussion and outlook}

One of the main issues that resulted in the number of constraints regarding the quality of vehicle data is that initially most of the automotive platforms were not designed to support data logging for datadriven evaluation. The development of a new automotive platform, supporting the new trend of datadriven design and assessment, is currently extremely costly. Thus, most of the automotive companies choose step-wise development of the existing platforms, adding up layers and layers of new lines of code to the existing one. The internal vehicle data and the external acquisition system are developed as intermediate solutions to support the current needs for data-driven evaluation. The analysis of the interviews revealed that both internal and external ways of vehicle data collection have their limitations. This is described in Table 3.

Table 3. Advantages and limitations of internal and external vehicle data

\begin{tabular}{|l|l|l|}
\hline Type of data & Advantages & Limitations \\
\hline $\begin{array}{l}\text { Internal } \\
\text { vehicle data }\end{array}$ & Supports system performance evaluation. & $\begin{array}{l}\text { Does not consider other systems in a complete } \\
\text { vehicle. }\end{array}$ \\
\cline { 2 - 3 } & $\begin{array}{l}\text { Provides data support in early stages of } \\
\text { ADAS verification. }\end{array}$ & $\begin{array}{l}\text { Provides poor data for driver behavior and } \\
\text { driving context evaluation (is not able to } \\
\text { deliver time-stamped data). }\end{array}$ \\
\cline { 2 - 3 } & $\begin{array}{l}\text { Allows collection of data from all company } \\
\text { vehicles. }\end{array}$ & $\begin{array}{l}\text { Changing the data requirements is difficult } \\
\text { since no changes are possible at the late stages. }\end{array}$ \\
\hline \multirow{2}{*}{$\begin{array}{l}\text { External } \\
\text { (ND data) }\end{array}$} & $\begin{array}{l}\text { Provides reliable data for context-aware } \\
\text { evaluation. }\end{array}$ & $\begin{array}{l}\text { Requires additional equipment and therefore } \\
\text { cannot be expanded to the complete vehicle } \\
\text { pool. }\end{array}$ \\
\cline { 2 - 3 } & $\begin{array}{l}\text { Helps to gain understanding of driver } \\
\text { behavior (allows identification of } \\
\text { behavioral patterns and applied use }\end{array}$ & $\begin{array}{l}\text { Professional customers might bring bias, } \\
\text { performing their tasks better. Real customers } \\
\text { need to agree to participate in the ND study and }\end{array}$ \\
\cline { 2 - 3 }
\end{tabular}




\begin{tabular}{|l|l|}
\hline scenarios). & share their data. \\
\hline The data can be easily reorganised and & \\
more data can be added to the evaluation & \\
process.
\end{tabular}

The internal data logging is not able to deliver time-stamped data and therefore can only be used for system performance verification; it is not able to support the context and driver behavior assessment. External ND data can overcome this limitation, providing reliable data for contextaware ADAS evaluation. However, to provide the required data every vehicle needs to be additionally instrumented with an external acquisition system. This does not allow the OEM to expand the study to the whole vehicle fleet of real users. In addition, GDPR standards do not allow the OEM to collect the data from customers without their consent, which significantly decreases customers' response rate. On the other hand, the OEM's employees who test instrumented vehicles bring a bias in user behavior evaluation, as they are often much more experienced in using support systems due to their work tasks and engineering background.

Another problem is that ADAS performance verification currently starts much earlier than the ND study. The main activities for system performance verification happen in the pre-production phase. When the ADAS gets to the production phase, only a follow up of the system is possible. The ND study for driver behavior evaluation is in contrast scheduled more heavily in the post-production phase. At the same time, the external ND data is more effective in providing comprehensive insights into how the driver interacts with the system in a context. Thus, with the appearance and quick development of ND data, the benefits of the use of internal vehicle data become questionable. If the external data collection would start earlier in the pre-production phase, developers could consider eventual issues with driver interaction with the ADAS and problems associated with system performance in different driver contexts.

A further issue depicted in the interviews is that the roles in the ADAS development team are separated. While developers are responsible for system performance verification, marketing focu ses on usage evaluation. Such a separate way of driver and system evaluation is not valid for systems such as ADAS, where there is a strong interrelation between the driver and the ADAS. Today, the methods used for system and driver evaluation are determined by traditions in the current OEM's practices, where the system performance is mostly evaluated by quantitative methods using objective data and driver behavior is evaluated by qualitative methods using predominantly subjective data. The authors believe there needs to be a re-evaluation of the adopted approach for ADAS evaluation. The methods used for ADAS evaluation need to be improved to support a holistic approach for ADAS evaluation that would consider the driver, the system, and the driving context altogether (Orlovska et al., 2020).

\section{Conclusions}

The aim of this study to understand the means and methods which are used for ADAS evaluation. The analysis of interviews' data revealed that the assessment of the ADAS performance without the acknowledgment of driver behavior and the driving context in a particular situation prevent developers from a comprehensive understanding of driver-system interaction. Thus, with the development of ND studies, where the external vehicle data has the potential to support a complex ADAS assessment, the use of internal vehicle data became questionable since it contributes only to the system performance evaluation. The authors believe that the main focus needs to be set on the further development of ND studies. The data signals that can be extracted from a vehicle needs to be further developed.

Another significant improvement can be made if the acquisition system used for external data collection will be integrated into all vehicles entering the market. Today it is too costly for the automakers. Finding a design solution to a cheaper acquisition system will allow OEMs to integrate the data acquisition system in the vehicle and to collect data from real drivers. This will help to eliminate the bias that professional customers can bring. 


\section{Acknowledgments}

The authors would like to express gratitude towards Sweden's Innovation Agency VINNOVA for the funding of the project, Grant no 2018-05017. We also want to thank all the participants without whom the study would not have been possible.

\section{References}

Ahlström, C. et al. (2018), "Effects of the road environment on the development of driver sleepiness in young male drivers", Accident Analysis \& Prevention, Vol. 112, pp. 127-134. https://doi.org/10.1016/j.aap.2018.01.012

Ahmed, M.M. and Ghasemzadeh, A. (2018), "The impacts of heavy rain on speed and headway behaviors: an investigation using the SHRP2 naturalistic driving study data", Transportation research part C: emerging technologies, Vol. 91, pp. 371-384. https://doi.org/10.1016/j.trc.2018.04.012

Angelini, M. et al. (2018), STEIN: Speeding up Evaluation Activities With a Seamless Testing Environment INtegrator. In EuroVis.

Beggiato, M. and Krems, J.F. (2013), "The evolution of mental model, trust and acceptance of adaptive cruise control in relation to initial information", Transportation research part F: traffic psychology and behaviour, Vol. 18, pp. 47-57. https://doi.org/10.1016/j.trf.2012.12.006

Benmimoun, M. et al. (2013), "eurofot: Field operational test and impact assessment of advanced driver assistance systems: Final results", Proceedings of the FISITA 2012 World Automotive Congress, Springer, Berlin, Heidelberg, pp. 537-547. https://doi.org/10.1007/978-3-642-33805-2_43

Carta, T., Paternò, F. and de Santana, V.F. (2011), "Web Usability Probe: A Tool for Supporting Remote Usability Evaluation of Web Sites", Lecture Notes in Computer Science, pp. 349-357, http://dx.doi.org/10.1007/978-3642-23768-3_29.

Fridman, L. et al. (2019), "MIT advanced vehicle technology study: Large-scale naturalistic driving study of driver behavior and interaction with automation", IEEE Access, Vol. 7, pp. 102021-102038.

ISO/PAS 21448. (2019), Road vehicles - Safety of the intended functionality. Available at: https://www. iso.org/standard/70939.html.

Leakkaw, P. and Panichpapiboon, S. (2018, December), "Real-Time Lane Change Detection Through Steering Wheel Rotation", 2018 IEEE Vehicular Networking Conference (VNC), IEEE, pp. 1-7. https://doi.org/ $10.1109 /$ vnc. 2018.8628461

Liang, Y. et al. (2016, October), "Considering traffic and roadway context in driver behavior assessments: a preliminary analysis", Adjunct Proceedings of the 8th International Conference on Automotive User Interfaces and Interactive Vehicular Applications, ACM, pp. 87-92. https://doi.org/10.1145/3004323.3004333

Mitrovic, D. (2005), "Reliable method for driving events recognition", IEEE transactions on intelligent transportation systems, Vol. 6 No. 2, pp. 198-205. https://doi.org/10.1109/tits.2005.848367

Neale, V.L. et al. (2005), An overview of the 100-car naturalistic study and findings, National Highway Traffic Safety Administration, Paper, 5, p. 0400.

NVivo 12. (2019), What is NVivo? Available at: https://www.alfasoft.com/en/products/statistics-andanalysis/nvivo.html (accessed 10.11.2019).

Orlovska, J. et al. (2019, July), Mixed-Method Design for User Behavior Evaluation of Automated Driver Assistance Systems: An Automotive Industry Case. Proceedings of the Design Society: International Conference on Engineering Design, Cambridge University Press, Vol. 1 No. 1, pp. 1803-1812. https://doi.org/10.1017/dsi.2019.186

Orlovska, J. et al. (2020), Effects of the driving context on the usage of Automated Driver Assistance Systems (ADAS)-Naturalistic Driving Study for ADAS evaluation, Transportation Research Interdisciplinary Perspectives, p. 100093.

Papazikou, E., Quddus, M.A. and Thomas, P. (2017), "Detecting deviation from normal driving using SHRP2 NDS data”, Presented at the Transportation Research Board (TRB) 96th Annual Meeting, January 8th-12th, 2017, Washington, DC, US.

SAE International. (2018), J3016_201806 - Taxonomy and Definitions for Terms Related to Driving Automation Systems for On-Road Motor Vehicles.

Yin, R.K. (2013), "Validity and generalization in future case study evaluations", Evaluation, Vol. 19 No. 3, pp. 321-332. https://doi.org/10.1177/1356389013497081

Zhai, Y. et al. (2018, June), A Context-Aware Evaluation Method of Driving Behavior. Pacific-Asia Conference on Knowledge Discovery and Data Mining, Springer, Cham, pp. 462-474. https://doi.org/10.1007/978-3319-93034-3_37 